\title{
ENTREVISTA
}

\section{La franc-maçonnerie à l'île Maurice de 1778 à 1915 : entre influences françaises et britanniques, la construction d'une identité mauricienne}

Sandra D. Brinda Venkaya Reichert a défendu une thèse doctorale en Études Anglophones sous le titre « La franc-maçonnerie à l'île Maurice de 1778 à 1915 : entre influences françai ses et britanniques, la construction d'une identité mauricienne » à l'université de BordeauxMontaigne, en France, le 26 juin 2017.

Sandra D. Brinda Venkaya Reichert est née en 1975 à l’Ile Maurice.

L'auteure nous a concédé l'interview suivant.

Pouvez-vous nous indiquer les raisons pour lesquelles vous avez choisi d'entreprendre votre recherche sur la Franc-maçonnerie?

La franc-maçonnerie s'est imposée comme une évidence dans mon cheminement universitaire au fil des années. Après trois mémoires de recherches universitaires sur les identités mauriciennes, autour de la littérature d'expression française, en traductologie (avec une orientation sociolinguistique du français régional mauricien) et enfin en civilisation britannique sur la contribution de 1'Eglise d'Angleterre dans la construction de la société devenue 'mauricienne', la franc-maçonnerie a été l'étape naturelle vers l'analyse approfondie d'une société qui n'a eu de cesse de se métamorphoser. En effet, l'élément déclencheur de ces recherches sur la franc-maçonnerie de cette thèse de doctorat fut que ces recherches, menées en amont, mirent en évidence la perplexité des identités mauriciennes. Cette thèse sur la franc-maçonnerie n'est, de ce fait, qu'un prolongement logique d'un autre aspect des identités insulaires d'un pays qui fut si souvent qualifié de colonie française sous le drapeau britannique au cours du XIX ${ }^{\mathrm{e}}$ siècle. Elle est, ainsi, la concrétisation de plusieurs années de recherches dans les archives maçonniques et générales européennes et mauriciennes qui, année après année, ont conforté l'idée de départ que la franc-maçonnerie mauricienne n'avait jamais, jusqu'ici, été présentée sous l'angle choisi. De ce fait, l'approche utilisée est de montrer que les identités mauriciennes peuvent être étudiées audelà des façades politiquement correctes de l'histoire générale, écrite principalement par les colonisateurs français et britanniques, ainsi que des habitants de la colonie alors 
britannique, avant les années 1970, puis par des Mauriciens après l'indépendance en 1968. Lors des premières années post-indépendantes, la mouvance, marquée par un nationalisme nouvellement acquis, fut de souligner les blessures de la colonisation, avant qu'une nouvelle génération d'historiens, de sociologues et d'écrivains prenne à bras le corps la valeur ajoutée d'une île qui est devenue une entité pluriethnique qui découle de traditions aussi bien africaines, asiatiques, européennes que locales, en amalgamant les diverses périodes aussi bien de colonisations que des décennies d'indépendance.

Quelles sont les sources que vous avez utilisées?

La méthodologie utilisée pour cette thèse fut de réunir plusieurs types de sources, aussi bien maçonniques, sociologiques, historiques, journalistiques que religieuses, afin d'affiner l'analyse de la société pluriethnique et cosmopolite et en utilisant, cette fois-ci, les réseaux maçonniques comme fil conducteur. Elle vise également à cerner les raisons d'une lacune historiographique qui est le manque d'études scientifiques sur la franc-maçonnerie. Les principales bibliothèques consultées en Europe pour les sources primaires sont la Library and Museum of Freemasonry, située au Freemasons' Hall de Londres; le Fonds maçonnique, hébergé par la Bibliothèque Nationale de France du site Richelieu à Paris; les archives du Grand Orient de France, rue Cadet à Paris, et les archives de La Grande Loge de France, rue Puteaux à Paris. Rebecca Hayes, l'archiviste actuelle de Grande Loge d'Irlande a également mis à disposition des documents de ses archives relatifs aux loges irlandaises qui furent implantées à Maurice. Quant aux archives des loges écossaises et de la Grande Loge d'Écosse, elles sont accessibles au Freemasons' Hall de Londres. Les ressources de ces bibliothèques ne sont pas, pour la plupart, accessibles à l'Ile Maurice. Des informations que certains historiens mauriciens n'avaient jamais exploitées jusqu'à présent le furent au cours de ces recherches. Elles ont permis de retracer le cheminement des maçons du XVIII ${ }^{\mathrm{e}}$ siècle jusqu'au début du $\mathrm{XX}^{\mathrm{e}}$ siècle, de connaître leurs attentes et leurs projets ainsi que de cerner leurs frustrations et les contraintes de la vie coloniale à travers leurs propres récits.

Concernant les sources secondaires, outre les bibliothèques citées plus haut, d'autres bibliothèques européennes et mauriciennes furent consultées tels que la British Library à Londres, la Bibliothèque François Mitterrand à Paris, les archives du Grand Orient de Bruxelles ainsi que les fonds documentaires mauriciens de la Bibliothèque Nationale de Maurice (à Port-Louis), de la Carnegie Library (à Curepipe) et des Mauritius National Archives. Des journaux maçonniques du XIX ${ }^{\mathrm{e}}$ siècle, tels que le Freemasons' Quarterly Magazine et le Masonic Mirror, ont été utilisés car ils couvraient les activités des loges britanniques à travers l'Empire britannique et publiaient des lettres de maçons qui étaient à l'étranger au XIX ${ }^{\mathrm{e}}$ siècle. A la National Library de Maurice, une grande partie des journaux 
du XIX ${ }^{\mathrm{e}}$ siècle (tels que Le Cernéen, The Merchants and Planters Gazette et Le Mauricien) sont accessibles et un long travail de recherche fut effectué pour retrouver les articles se référant à la franc-maçonnerie puisqu'il n'y a pas de catalogue les répertoriant. Pour compléter le travail de terrain, les écrits des historiens et maçons contemporains furent utilisés. Il y a un nombre restreint de livres et d'articles consacrés à la franc-maçonnerie mauricienne après le XIX ${ }^{\mathrm{e}}$ siècle et l'approche scientifique d'un tel sujet est encore assez peu répandue.

Quelles furent les principales difficultés auxquelles vous vous êtes confrontées ? Comment les avez-vous surmontées?

La fiabilité des sources a souvent été un obstacle car une grande majorité des écrits par les francs-maçons eux même depuis le XVIIIIe siècle a dû être contrevérifié et souvent avec le constat que certaines informations n'étaient soit pas vérifiables, car non référencées, soit incorrectes. Il est important de souligner la part d'idéalisation ou de subjectivité de certains maçons qui, en faisant l'historique de leur loge, se fondèrent grandement sur les détails biographiques les plus avantageux, sur les moments prestigieux et sur des descriptions poétiques au détriment de la situation réelle de la colonie. En effet, ces récits firent souvent abstraction des événements peu valorisants mais primordiaux, tout en ayant des positionnements très orientés sur d'autres thèmes. De plus, la préservation des archives des loges, comme les planches tracées depuis le XVIII ${ }^{\mathrm{e}}$ siècle, ne fut pas toujours une priorité à l'époque coloniale. En effet, le manque de préservation des travaux et de leur diffusion aux autres maçons, et éventuellement aux non-initiés, fut une lacune constatée au cours de ces recherches. Quand cela fut le cas, certaines erreurs historiques qui se transféraient oralement purent parfois être rectifiées. Il est incontestable que l'utilisation de ces archives primaires et secondaires comporte des inconvénients à plusieurs niveaux. Par exemple, l'impossibilité de retracer des dates exactes devient aussi une pierre d'achoppement quand certains auteurs confondent les dates du calendrier maçonnique à celles du calendrier grégorien ou mettent en avant des dates sans les justifier. Dans les manuscrits des loges françaises, le calendrier maçonnique est le plus souvent utilisé alors que ce ne fut pas le cas des manuscrits venant des loges anglaises. Un autre obstacle fut de constater la perte ou la destruction de certaines archives suite aux catastrophes naturelles, aux incendies ou, tout simplement, due à des erreurs humaines.

Quelles sont les principales problématiques historiques que votre travail a résolues?

Ce travail a mis en lumière la question des identités mauriciennes comme étant étroitement liée simultanément aux influences européennes et aux facteurs insulaires. Les principales 
problématiques furent, ainsi, de montrer comment la maçonnerie mauricienne s'est construite à la croisée des cultures coloniales française et britannique mais également face aux enjeux inattendus (géographiques et climatiques), face à ses liens avec une société cosmopolite sous tension socio-politique et face à un œcuménisme franco-britannique, constamment réévalué dû aux exigences des questionnements religieux et institutionnels des obédiences européennes. En effet, outre les liens étroits entre la franc-maçonnerie et les élites socio-économiques, cette thèse montre que d'autres relations ont façonné la maçonnerie locale, telles que les liens entre les loges et les religions (en particulier le Catholicisme romain); les liens avec le pouvoir administratif colonial, et ceux avec la société coloniale dans son ensemble. Certaines relations qui, de nos jours, sont oblitérées ou restent tabous, ne l'étaient pas au cours du XIX ${ }^{\mathrm{e}}$ siècle. Un des exemples de la visibilité de la maçonnerie fut la fréquence des articles des journaux locaux qui traitaient de ses célébrations et même des problèmes rencontrés par les maçons locaux. Certains aspects atypiques de ces identités sont mis en avant dans cette thèse tels que les cérémonies maçonniques et religieuses de plusieurs traditions lors des célébrations de la St Jean où les maçons défilaient avec leurs décorations et drapeaux dans la capitale, Port-Louis, avant de se rendre à la cathédrale anglicane où une cérémonie religieuse était célébrée par le chapelain des troupes lui-même en tenue maçonnique. Ces histoires croisées montrent que la franc-maçonnerie avait une visibilité qui fut aussi bien avantageuse pour elle mais a aussi apporté son déclin. Par exemple, alors que depuis la fin du XVIII ${ }^{\mathrm{e}}$ siècle, la maçonnerie française du Grand Orient de France s'était développé dans une colonie française, elle dut s'adapter au changement de drapeau et elle ouvrit les portes de ses temples aux Britanniques dès les années 1810. Outre la fraternité maçonnique cultivée, les temples devinrent également un refuge hors de la société coloniale en conflits, réunissant des profils, qui dans le civil, s'adonnaient à des rivalités : les anciens colons français, les nouveau colonisateurs britanniques et les gens de couleur qui cherchaient une place dans une société gangrénée par la ségrégation raciale. Cette thèse montre, de ce fait, que la maçonnerie mauricienne fut souvent une soupape lors des périodes de tensions car elle arriva à réunir des personnalités qui œuvraient dans des cercles politiques, économiques et culturels différents. Dans ce carrefour des identités, la maçonnerie ne put que se transformer. Les aspects insulaires, qui entraient en jeu à la croisée des cultures coloniales française et britanniques, montrèrent que, malgré la richesse des relations entre les différentes nationalités, la fraternité universelle avait ses limites. Les faiblesses de la maçonnerie furent nombreuses comme le manque de rigueur dans l'administration des loges, le manque de mixité ethnique, l'ethnocentrisme, le manque de tolérance et même un certain affairisme. Cette thèse montre, ainsi, que la maçonnerie est aussi une association d'hommes (car les femmes n'étaient pas admises dans les différentes obédiences présentes) qui étaient les produits de leur temps et étaient, parfois, les reflets des discriminations et des 
inégalités de la société coloniale dans laquelle ils vivaient. Les différents conflits politiques (dans une société où l'abolition de l'esclavage, les revendications de représentativité politique des non-britanniques, l'antimaçonnisme et une rébellion pour accéder à la décolonisation) eurent, inévitablement, des effets néfastes sur les relations maçonniques. Les conséquences, surtout à la fin du $\mathrm{XIX}^{\mathrm{e}}$ siècle et au début $\mathrm{du} \mathrm{XX}^{\mathrm{e}}$ siècle, après presqu'un siècle d'entente malgré les frictions, furent multiples : le retrait sur soi ou sur sa communauté par certains maçons; le choix de la famille au détriment de la maçonnerie pour éviter le jugement de la société coloniale; l'apologie d'un nationalisme mauricien; la fin du partage des temples maçonniques par plusieurs obédiences, et une rivalité entre les loges.

S'il vous plaît, pourriez-vous résumer l'essence de votre thèse en deux lignes?

Cette thèse retrace les étapes de l'implantation de la franc-maçonnerie de traditions françaises puis britanniques dans la colonie et met en lumière le fait que les loges qui se développèrent, souvent en autarcie, dans cette île de l'Océan indien, eurent une histoire riche de par le fait qu'elles furent créées loin des métropoles par des expatriés, mais également par des colons, devenus au fil des décennies, les enfants du sol. Les loges maçonniques firent de ce fait une double expérience : continuer à préserver la pureté des traditions des obédiences européennes tout en subissant une créolisation identitaire.

Quelles sont les leçons, à tous les niveaux, personnelles et professionnelles, que vous avez déduites de votre expérience de recherche?

La rigueur fut le maitre-mot de ce cheminement de recherches et de rédaction. Un travail sur soi est forcément aussi devenu une priorité afin de conserver une approche scientifique.

Et maintenant, quels sont vos projets professionnels?

Après presque vingt ans dans les domaines de l'enseignement et de la formation professionnelle, $\mathrm{j}$ 'ai entamé un changement de carrière qui me procure un avantage certain : la possibilité de continuer mes recherches sans pression. Je suis devenue directrice éditoriale.

Voulez-vous mettre en exergue des facteurs qui n'ont pas été contemplés dans cette entrevue et que vous voudriez partager?

Il est important de souligner le fait qu'une des conclusions de cette thèse fut de montrer que la maçonnerie mauricienne, bien que n'impliquant que quelques centaines de maçons 
annuellement et même si elle s'est développée géographiquement loin des métropoles, a eu une importance réelle dans le paysage maçonnique européen et mondial. La maçonnerie mauricienne, tout en gardant des liens étroits avec les obédiences, a prouvé sa différence en préservant les rites et traditions européennes dans une société pluriethnique alors même que beaucoup de cartes du monde de l'époque ne plaçaient pas l'île dans le giron de l'Empire britannique et que certaines ne la mentionnait même pas.

Cet interview s'est réalisé de façon numérique le 2 octobre 2017.

Les auteurs de 1'entrevue: Ricardo Martínez Esquivel et Yván Pozuelo Andrés, Directeur et Éditeur REHMLAC+, doi : https://doi.org/10.15517/rehmlac.v9i2.31262 